\title{
FLOW USING FEW EDGES IN A BIPARTITE NETWORK
}

\author{
THINH D. NGUYEN
}

\section{Problem statement}

If we have a set of sources $S$ with total capacity $C$ and a set of $\operatorname{sinks} T$ with the same capacity $C$, but not necessarily the same cardinality, our problem MinEDGEFLOW asks whether there an efficient way to find the minimum number of direct edges between sources and sinks and their required capacity, assuming flow $=C$.

Claim 1. We have that Partition $\leq_{p}$ MinEdgeFlow

In this claim, the hardness of PARTITION is known since [1]. Now, we prove our claim.

Proof. Consider a partition problem instance with input $a_{1}, \ldots, a_{n}$. We create a complete bipartite graph with 2 source vertices each with capacity $\frac{1}{2} \sum_{i=1}^{n} a_{i}$, and $n$ sink vertices, where the $i$ th vertex has capacity $a_{i}$. Each edge has infinite capacity.

It is easy to verify there is a partition with equal sum if and only if the solution to our problem on $G$ has $n$ edges.

\section{Conclusion}

As long as we study a mathematical conjecture, we should encourage ourselves of having enough labouring hours on popular maths books. Then, reading some articles on theory of computing like [3] is a good practice. Only after that, could we think of the ultimate final for all mathematics sciences.

\section{REFERENCES}

1. Michael R. Garey, David S. Johnson, Computers and Intractability: A Guide to the Theory of NP-Completeness

2. David S. Johnson, The NP-Completeness Column: An Ongoing Guide. pp.393-405

3. Phan Dinh Dieu, Le Cong Thanh, Le Tuan Hoa, Average Polyno-mial Time Complexity of Some NP-Complete Problems, Theor. Comput. Sci. 46(3): pp.219-237 (1986)

Current address: Department of Mathematics, Moscow State University

Email address: kosmofarmer@yandex.com

Key words and phrases. bipartite, edge, flow.

Perebor. 